\title{
Conducción hopping en películas nanocristalinas del compuesto CZTSe usado como capa absorbente en celdas solares
}

\author{
N. Sena ${ }^{\varpi}$, H. M. Mateus, A. Dussan
}

Hopping conduction in nanocrystalline composite films of CZTSe used as solar cell absorber layer

\section{Abstract}

Here, we present electronic and transport properties of quaternary $\mathrm{Cu}_{2} \mathrm{ZnSnSe}_{4}$ (CZTSe) nanocrystalline films fabricated by physical co-evaporation. The samples were grown on soda-lime glass substrates and synthesis parameter ranges, $\mathrm{Cu}$ mass and substrate temperature were varied. Using thermopower at room temperature and spectral transmittance we found that the material is characterized by n-type conductivity and forbidden energy bandwidth of $1.7 \mathrm{eV}$, respectively. Electrical conductivity means (low temperature region; 90-200 K) showed that conductivity processes occur via variable range hopping between extended states. We obtained the parameters characterizing this mechanism, activation energy $\left(W_{\text {hopp }}\right)$, and range hopping $\left(R_{h o p p}\right)$, by employing the percolation theory and diffusion model. The density of defect states near the Fermi level of the material, $\mathrm{N}\left(\mathrm{E}_{\mathrm{F}}\right)$ of the CZTSe samples is about $3,403 \times 10^{18} \mathrm{~cm}^{-3} \mathrm{eV}^{-1}$. We found a correlation between deposition parameters and electrical properties and observed a parameter influence on the formation of additional phases in the compound.

Keywords: Hopping; transport properties; semiconductors.

Edited by Beynor Paez $\square$ \& Alberto Acosta

Departamento de Física, Grupo de Materiales Nanoestructurados, Universidad Nacional de Colombia - Bogotá, Colombia.

Received: 22-11-2013 Accepted: 01-04-2014

Published on line: 08-05-2014

Citation: Seña N, Mateus HM, Dussan A (2014) Conducción hopping en películas nanocristalinas del compuesto CZTSe usado como capa absorbente en celdas solares. Universitas Scientiarum 19(2): 147-152 doi: 10.11144/Javeriana.SC19-2.chpn

Funding: Universidad Nacional de Colombia - DIB.

Electronic supplementary material: NA

\section{Introducción}

Numerosos estudios han centrado su interés en el desarrollo de celdas solares (Islam et al. 2009, Rousset et al. 2011, Nerat 2012, Wei et al. 2013) basadas en los compuestos CdTe y Cu (InGa), (S, Se2), (CIGS). Para celdas tipo CIGS y CdTe en películas delgadas, se han reportado valores en la eficiencia de conversión, esto es alrededor del 20 y 15\% para celdas CIGS y CdTe respectivamente (Repins et al. 2008, Kranz et al. 2012, Nerat 2012, Kranz et al. 2013, Powalla et al. 2013). Sin embargo, la búsqueda de nuevos materiales que actúen como capa absorbente se han centrado en precursores que sean abundantes en la naturaleza $\mathrm{y}$ sin incidencia con el medio ambiente (Tsega et al. 2013). Dentro de las propuestas para sustituir 
estos materiales sin comprometer la eficiencia se reportan los compuestos cuaternarios $\mathrm{Cu}_{2} \mathrm{ZnSnSe}_{4}$ (CZTSe) y $\mathrm{Cu}_{2} \mathrm{ZnSnS}_{4}$ (CZTS) como materiales promisorios y potencialmente importantes en la fabricación de dispositivos fotovoltaicos (Juškènas et al. 2012, Gurav et al. 2014). En la actualidad los estudios alrededor de los compuestos CZT(S,Se) han enfatizado sobre la optimización en los parámetros de síntesis y el conocimiento de sus propiedades estructurales y morfológicas (Chen et al. 2013, Mousel et al. 2013, Redinger et al. 2013), así como también su implementación en celdas solares tipo laboratorio (Sun et al. 2011, Guo et al. 2012, Todorow et al. 2013).

En la actualidad no se encuentran reportes de las propiedades eléctricas ni de los mecanismos de transporte que gobiernan al material que soporten el uso de estos compuestos como capa absorbente en dispositivos fotovoltaicos. Recientemente, se ha mostrado que las eficiencias de conversión son prometedoras gracias a las propiedades eléctricas que favorecen el transporte (Ilari et al. 2012, Fella et al. 2013, Tsega et al. 2013).

Se presentan resultados experimentales de los posibles mecanismos de transporte eléctrico en el compuesto $\mathrm{Cu}_{2} \mathrm{ZnSnSe}_{4}$, preparado por la técnica PVD (Physical Vapor Deposition) en varias etapas. Se realizaron medidas de conductividad a oscuras en función de la temperatura. Se establece que el transporte para la región de alta temperatura se realiza vía activación térmica de los portadores, mientras que para la región de temperaturas por debajo de los $300 \mathrm{~K}$ el transporte es gobernado por hopping de rango variable (VRH). Para completa caracterización eléctrica y teniendo en cuenta los fundamentos de la teoría de percolación, fue posible la determinación de los parámetros de hopping: energía de activación hopping $\left(\mathrm{W}_{\text {hopp }}\right)$ y rango hopping $\left(\mathrm{R}_{\text {hopp }}\right)$.

\section{Materiales y métodos}

Las películas delgadas de CZTSe fueron fabricadas por el método de co-evaporación en tres etapas en una cámara a alto vacío en atmósfera de selenio. Se variaron parámetros de síntesis: temperatura del sustrato durante la etapa de evaporación del $\mathrm{Cu}$ $\left(\mathrm{T}_{\mathrm{S}}\right)$ entre 573 y $773 \mathrm{~K}$ y la masa del $\mathrm{Cu}(\mathrm{M})$ como material precursor en la proporción dada por la relación $\mathrm{Cu} /(\mathrm{ZnSe}+\mathrm{Sn})$. Dicha proporción fue de $0,416-0,472-0,524$ con una relación constante Zn/ Sn de 1.577. Se depositaron contactos eléctricos de plata según la geometría requerida y posteriormente aplicada una diferencia de potencial de $5 \mathrm{mV} / \mathrm{cm}$ durante los procesos de medición. Las medidas de conductividad a oscuras $\left(\sigma_{\text {OSC }}\right)$ se realizaron en el rango de temperaturas entre 90 y $400 \mathrm{~K}$, utilizando el método de las cuatro puntas según la geometría de Van Der Pauw. Las medidas fueron realizadas en un equipo PPMS Resistivity y la corriente fue medida con un electrómetro Keithley 617 conectado a un sistema de adquisición de datos.

Se realizaron medidas de termopotencia a presión atmosférica y se estableció que las muestras presentaron una conductividad tipo n. A partir de medidas de perfilometría se encontró que el espesor promedio de las películas fue de $700 \mathrm{~nm}$.

\section{Resultados}

Las curvas de $\sigma_{\mathrm{OSC}}$ en función de $\mathrm{T}$ para las películas delgadas del compuesto $\mathrm{Cu}_{2} \mathrm{ZnSnSe}_{4}$ se obtuvieron para el conjunto de muestras de CZTSe variando Ts y $\mathrm{M}$ del cobre durante el depósito (Figura 1a) y se observa el comportamiento de la conductividad gobernado por dos regiones diferentes de transporte que no pueden ser ajustados por una misma función lineal en el marco de un gráfico Arrhenius.

Se presentan las medidas de conductividad a oscuras obtenidas, pero en esta ocasión, en función de $\mathrm{T}^{-1 / 4}$ (Figura 1b). Se observa que todas las curvas pueden ser ajustadas con una función lineal que identifican el mecanismo de transporte de portadores como hopping de rango variable (VRH).

Por otra parte, un aumento de la $\mathrm{T}_{\mathrm{S}}(\mathrm{Cu})$ se traduce en una disminución de la conductividad en la región de bajas temperaturas asociada al incremento de los estados de defectos cerca del nivel de Fermi en el material (Figura 3).

\section{Discusión}

El aumento en $\sigma_{\mathrm{OSC}}$ (Figura 1a) es asociado a la presencia de portadores de carga en el material debido al incremento en la masa del $\mathrm{Cu}$. Sin embargo, cuando se aumenta nuevamente la masa del $\mathrm{Cu}$ de 0,117 a 
0,131 g no se observa mayor valor en la conductividad, el cual puede ser asociado a una disminución en la movilidad eléctrica por acomodación de los átomos de $\mathrm{Cu}$ en los intersticios de la red; generando defectos estructurales y posibles dislocaciones.

El comportamiento eléctrico descrito anteriormente se estudió en dos regiones de temperatura bien definidas, a altas temperaturas, esto es, por encima de temperatura ambiente y, la región de bajas temperaturas cercanas a los $100 \mathrm{~K}$. Se observa que para la región de $\mathrm{T}>300 \mathrm{~K}$ las medidas de conductividad presentan un comportamiento tipo Arrhenius identificándose el transporte de portadores por activación térmica. A partir de la relación log $\sigma_{\mathrm{OSC}} \mathrm{vs} 1000 / \mathrm{T}$, se encontraron los valores para las energías de activación $\mathrm{E}_{\mathrm{a}}(0,337$ $\mathrm{eV} ; 0,028 \mathrm{eV} ; 0,041 \mathrm{eV}$ ) para las muestras con $\mathrm{M}(\mathrm{Cu})$ de 0,118 g, 0,104 g y 0,131 g respectivamente. Si bien, se observa disminución en la energía de activación cuando la masa del $\mathrm{Cu}$ aumenta $(\mathrm{M}(\mathrm{Cu})=0,131 \mathrm{~g})$, la proporción de $\mathrm{Se} /(\mathrm{Cu}+\mathrm{Se})$ aumenta, con lo cual se enlaza con mayor facilidad con el $\mathrm{Cu}$ modificando los estados de valencia del $\mathrm{Cu}$ y favoreciendo la transferencia de carga entre estos estados de valencia (Liu et al. 2008, Wandekar et al. 2009).
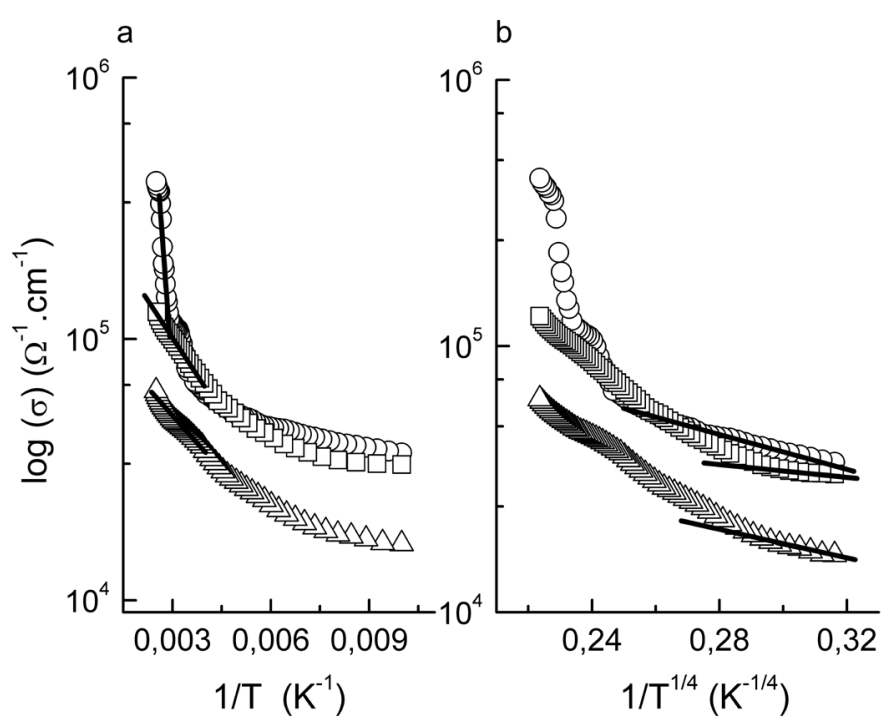

Fig. 1. Conductividad a oscuras en función de la temperatura para muestras de $\mathrm{Cu}_{2} \mathrm{ZnSnSe}_{4}$ variando la $\mathrm{M}(\mathrm{Cu})$. a. Comportamiento Arrhenius par la región de T >300 K. b. Ajuste lineal Ecuación de Mott, evidencia Hopping de Rango Variable. $(\Delta$ $\mathrm{M}(\mathrm{Cu})=0,104 \mathrm{~g}, \mathrm{O} \mathrm{M}(\mathrm{Cu})=0,118 \mathrm{~g}, \square \mathrm{M}(\mathrm{Cu})=$ 0,131 g. Las líneas rectas representan los ajustes para el modelo de Arrhenius y VRH respectivamente).
El mecanismo de VRH, observado en la curva de conductividad para la región de bajas temperaturas (Figura 1b) se identifica por la posibilidad que tienen los portadores de ocupar estados energéticos más cercanos en comparación con aquellos cuya distancia entre estados sea pequeña. Para mayor confianza, que este mecanismo es el más apropiado, a través de la teoría de percolación, se calcularon los parámetros hopping ( $\mathrm{R}_{\text {hopp }}$ : Rango hopping, $\mathrm{W}_{\text {hopp }}$ : Energía de activación hopping) que caracterizan el transporte VRH (Tabla 1).

Tabla 1. Valores de los parámetros hopping para el mecanismo VRH obtenidos para el compuesto CZTSe variando $\mathrm{M}(\mathrm{Cu})$ y la $\mathrm{T}_{\mathrm{S}}$ usando la teoría de percolación.

\begin{tabular}{cccc}
\hline & & \multicolumn{2}{c}{ Modelo Percolaión } \\
$\mathbf{C u}_{2} \mathbf{Z n S n S e}_{4}$ & $\mathbf{W}_{\text {hopp }}(\mathbf{c m})$ & $\mathbf{R}_{\text {hopp }}(\mathrm{eV})$ \\
\hline & 573 & $6,03 \times 10^{-7}$ & 0,0054 \\
\hline $\mathbf{T}_{\mathbf{s}}(\mathbf{C u})(\mathbf{K})$ & 673 & $2,29 \times 10^{-6}$ & 0,0205 \\
& 773 & $1,24 \times 10^{-6}$ & 0,0111 \\
& 0,104 & $1,76 \times 10^{-7}$ & 0,0432 \\
\hline $\mathbf{M}(\mathbf{C u})(\mathbf{g})$ & 0,118 & $1,74 \times 10^{-7}$ & 0,0429 \\
& 0,131 & $1,90 \times 10^{-7}$ & 0,0468 \\
\hline
\end{tabular}

A partir de los valores obtenidos para los parámetros hopping, se observa, que las energías obtenidas para $W_{\text {hopp }}$ ratifican que el ancho de banda alrededor de $\mathrm{E}_{\mathrm{Fermi}}$, es responsable de la conducción para el mecanismo de VRH (angosto a bajas temperaturas; Tabla 1); lo cual es una condición dada por la teoría de percolación. Lo anterior se evidencia tanto para las muestras de CZTSe variando la masa como la temperatura de $\mathrm{Cu}$.

Cuando se aumenta la masa del $\mathrm{Cu}$ pasando de 0,118 g a 0,131 g se encuentra que $\mathbb{W}_{\text {hopp }}$ disminuye aunque no en la misma proporción como para una disminución de la $\mathrm{M}(\mathrm{Cu})$ desde 0,118 a 0,104 g. En este caso la disminución de $\mathrm{W}_{\text {hopp }}$ es atribuida a la 
redistribución del $\mathrm{Cu}$ en exceso en la estructura, esta reacomodación posiblemente facilite el transporte de saltos entre vecinos cercanos energéticamente.

Se reporta la energía de activación hopping observada para cada uno de los casos (Figura 2), variando la $\mathrm{M}(\mathrm{Cu})$ y TS $(\mathrm{Cu})$.

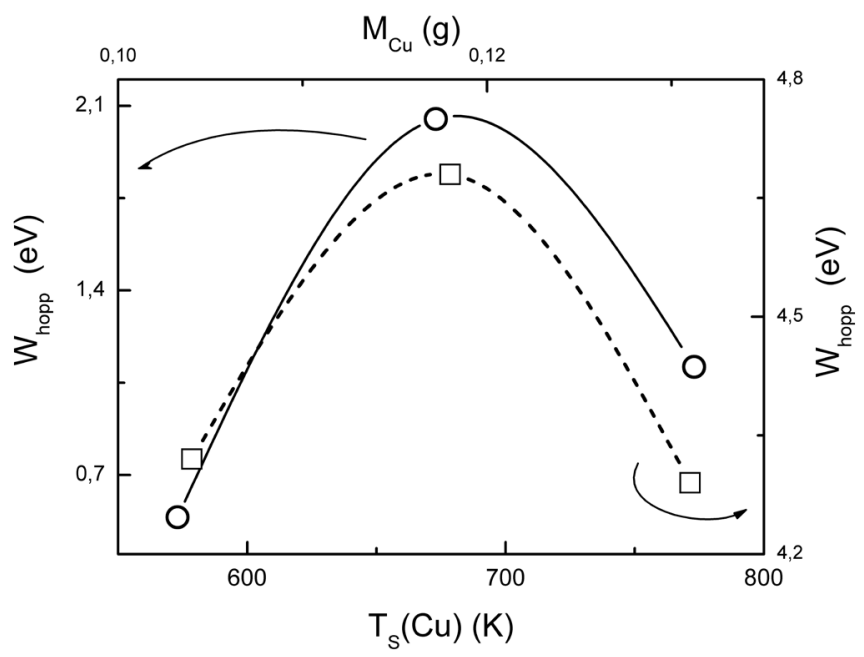

Fig. 2. Energía de activación hopping como resultado de la variación en la masa de $\mathrm{Cu}$ y la temperatura del sustrato durante la evaporación del $\mathrm{Cu}\left(\mathrm{O}\right.$ representa la $\mathrm{W}_{\text {hopp }} \mathrm{Vs} \mathrm{M}(\mathrm{Cu}), \square$ representa la $\mathrm{W}_{\text {hopp }} \mathrm{Vs} \mathrm{M}(\mathrm{Cu})$ Las líneas continuas y punteadas son ayuda para la visualización de la tendencia de $W_{\text {hopp }}$ en cada caso.

Un aumento de la $\mathrm{W}_{\text {hopp }}$ seguido de una disminución en dicho valor con el aumento tanto de la masa como la temperatura del sustrato durante la evaporación del $\mathrm{Cu}$ se pudo observar en las medidas obtenidas para los parámetros hopping (Figura 2). Este hecho es indicativo que ratifica el VRH como mecanismo de transporte para la región de bajas temperaturas en el compuesto CZTSe.

Otra manera de corroborar el VRH como mecanismo de transporte en nuestro material, es obteniendo una tendencia lineal en las curvas de resistencia en función de $T^{-1 / 4}$ (Taele et al. 2008, Shi et al. 2014). Lo anterior fue evidenciado con las muestras del cuaternario CZTSe cuando se varió la $\mathrm{T}_{\mathrm{S}}(\mathrm{Cu})$. Se presenta el logaritmo natural de la resistencia de las muestras cuaternarias en función de $\mathrm{T}^{1 / 4}$ (Figura 3), cuando variamos la $\mathrm{T}_{\mathrm{S}}(\mathrm{Cu})$.
El comportamiento lineal del $\operatorname{Ln}(\mathrm{R})$ Vs $1 / \mathrm{T}^{1 / 4}$ para el compuesto CZTSe (Figura 3) demuestra que la posibilidad de transporte eléctrico en esta región de T ocurre mediante saltos entre estados ubicados cerca del nivel de Fermi y con una densidad constante para la misma región. Lo anterior se traduce en transporte vía hopping de rango variable.

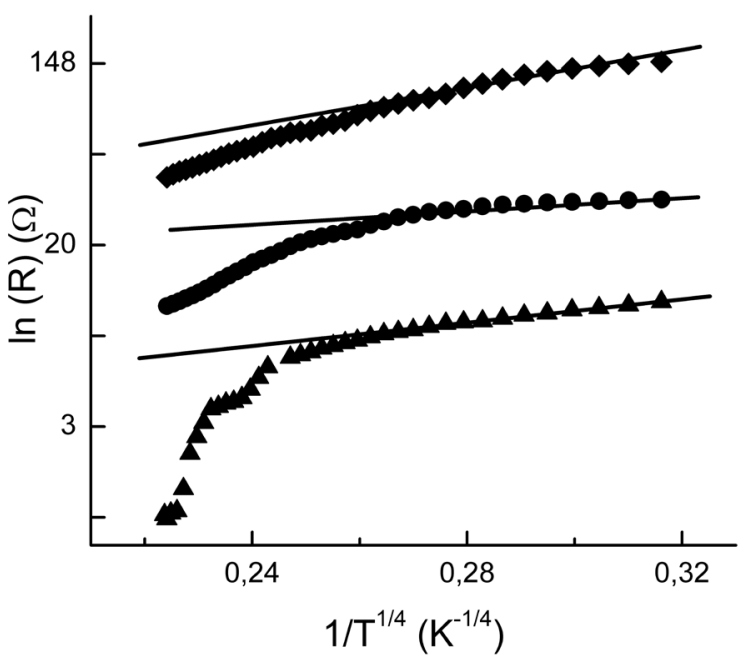

Fig. 3. $\operatorname{Ln}(\mathrm{R}) \mathrm{Vs} 1 / \mathrm{T}^{1 / 4}$ para el compuesto CZTSe cuando se varío la $\mathrm{T}_{\mathrm{s}}(\mathrm{Cu})$. Las líneas rectas continuas representan el ajuste lineal para un amplio rango de T. $\left(\boldsymbol{\bullet} \mathrm{T}_{\mathrm{s}}(\mathrm{Cu})=573 \mathrm{~K}, \boldsymbol{\Delta} \mathrm{T}_{\mathrm{s}}(\mathrm{Cu})=673 \mathrm{~K}, \bullet \mathrm{T}_{\mathrm{s}}(\mathrm{Cu})=\right.$ $773 \mathrm{~K})$.

\section{Conclusión}

A partir de medidas de conductividad en función de la temperatura, se encontró que el compuesto cuaternario $\mathrm{Cu}_{2} \mathrm{ZnSnSe}_{4}$ es gobernado por dos mecanismos de transporte eléctrico dependiendo de la región de $\mathrm{T}$ analizada. Para la región de $\mathrm{T} \geq 300 \mathrm{~K}$, se encontró que el mecanismo de transporte predominante es el de portadores térmicamente activados mientras que, para temperaturas menores, el transporte es gobernado por hopping de rango variable, VRH. La variación de los parámetros que caracterizan el transporte hopping presentan una tendencia similar con el aumento tanto de la masa como la temperatura del sustrato para el $\mathrm{Cu}$; siendo aún más probable el VRH para el transporte de portadores a $\mathrm{T}<100 \mathrm{~K}$. 


\section{Agradecimientos}

Este trabajo fue soportado por proyectos de la Universidad Nacional de Colombia - DIB. Agradecimiento al Grupo de Materiales Semiconductores y Energía Solar, por el apoyo en el laboratorio para preparación de las muestras.

\section{Conflicto de intereses}

Este trabajo no presenta conflicto de intereses.

\section{Referencias}

Chen S, Walsh A, Gong XG, Wei SH (2013) Classification of Lattice Defects in the Kesterite $\mathrm{Cu}_{2} \mathrm{ZnSnS}_{4}$ and $\mathrm{Cu}_{2} \mathrm{ZnSnSe}_{4}$ Earth-Abundant Solar Cell Absorbers. Advanced Materials 25:1522-1539 doi: 10.1002/ adma.201203146

Fella CM, Romanyuk YE, Tiwari AN (2013) Technological status of $\mathrm{Cu}_{2} \mathrm{ZnSn}(\mathrm{S}, \mathrm{Se})_{4}$ thin film solar cells. Solar Energy Materials \& Solar Cells 119:276-277 doi: 10.1016/j. solmat.2013.08.027

Guo Q, Ford GM, Yanga WC, Hagesa CJ, Hillhouse HW et al. (2012) Enhancing the performance of CZTSSe solar cells with Ge alloying. Solar Energy Materials \& Solar Cells 105:132-136 doi: 10.1016/j.solmat.2012.05.039

Gurav KV, Shin SW, Patil UM, Deshmukh PR, Suryawanshi MP et al. (2014) $\mathrm{Cu}_{2} \mathrm{ZnSnS}_{4}$ (CZTS)-based room temperature liquefied petroleum gas (LPG) sensor. Sensors and Actuators B: Chemical 190:408-413 doi: 10.1016/j.snb.2013.08.064

Ilari GM, Fella CM, Ziegler C, Uhl AR, Romaniuk YE et al. (2012) $\mathrm{Cu}_{2} \mathrm{ZnSnSe} 4$ solar cell absorbers spin-coated from amine-containing ether solutions. Solar Energy Materials \& Solar Cells 104:125-130 doi: 10.1016/j. solmat.2012.05.004

Islam MM, Ishizuka S, Yamada A, Sakurai K, Niki S et al. (2009) CIGS solar cell with MBE-grown ZnS buffer layer. Materials and Solar Cells 93:970-972 doi: 10.1016/j. solmat.2008.11.047

Juškėnas R, Kanapeckaite S, Karpavičienè V, Mockus Z, Pakštas V et al. (2012) A two-step approach for electrochemical deposition of $\mathrm{Cu}-\mathrm{Zn}-\mathrm{Sn}$ and $\mathrm{Se}$ precursors for CZTSe solar cell. Solar Energy Materials \& Solar Cells 101:277-282 doi: 10.1016/j.solmat.2012.02.007

KranzL, BuechelerS, Tiwari AN (2013) Technological status of CdTe photovoltaics. Solar Energy Materials \& Solar Cells 119:278-280 doi: 10.1016/j.solmat.2013.08.028

Kranz L, Perrenoud J, Pianezzi F, Gretener C, Rossbach P et al. (2012) Effect of sodium on recrystallization and photovoltaic properties of CdTe solar cells. Solar Energy Materials \& Solar Cells 105:213-219 doi: 10.1016/j. solmat.2012.06.019
Liu HQ, Song Y, Zhang SN, Zhao XB, Wang FP (2008) Thermoelectric Properties of $\mathrm{Ca}_{3-\mathrm{x}} \mathrm{Y}_{\mathrm{x}} \mathrm{CO}_{4} \mathrm{O}_{+} 9_{+\delta}$ ceramics. Journal of Physics and Chemistry of Solids 70:600603 doi: 10.1007/s10853-008-2990-6

Mousel M, Redinger A, Djemour R, Arasimowicz M, Valle V et al. (2013) $\mathrm{HCl}$ and $\mathrm{Br}_{2}-\mathrm{MeOH}$ etching of $\mathrm{Cu}_{2} \mathrm{ZnSnSe}_{4}$ polycrystalline absorbers. Thin Solid Films 535:83-87 doi: 10.1016/j.tsf.2012.12.095

Nerat M (2012) Copper-indium-gallium-selenide (CIGS) solar cells with localized back contacts for achieving high performance. Solar Energy Materials \& Solar Cells 104:152-158 doi: 10.1016/j.solmat.2012.05.020

Powalla M, Jackson P, Witte W, Hariskos D, Paetel S et al. (2013) High-efficiency $\mathrm{Cu}(\mathrm{In}, \mathrm{Ga}) \mathrm{Se}_{2}$ cells and modules. Solar Energy Materials \& Solar Cells 119:51-58 doi: 10.1016/j.solmat.2013.05.002

Redinger A, Mousel M, Djemour R, Gütay L, Valle N et al. (2013) $\mathrm{Cu}_{2} \mathrm{ZnSnSe}_{4}$ thin film solar cells produced via co-evaporation and annealing including a $\mathrm{SnSe}_{2}$ capping layer. Prog. Photovoltaics: Research and Applications 22:5157 doi: 10.1002/pip.2324

Repins I, Contreras MA, Egaas B, DeHart C, Scharf J et al. (2008) 19.9\%-efficient ZnO/CdS/CuInGaSe 2 solar cell with $81.2 \%$ fill factor. Progress in Photovoltaics 16:235-239 doi: $10.1002 /$ pip. 822

Rousset J, Donsanti F, Genevée P, Renou G, Lincot D (2011) High efficiency cadmium free $\mathrm{Cu}(\mathrm{In}, \mathrm{Ga}) \mathrm{Se}_{2}$ thin film solar cells terminated by an electrodeposited front contact. Solar Energy Materials \& Solar Cells 95:1544-1549 doi: 10.1016/j.solmat.2010.12.009

Shi C, Qin H, Zhao M, Wang X, Li L et al. (2014) Investigation on electrical transport, $\mathrm{CO}$ sensing characteristics and mechanism for nanocrystalline $\mathrm{La}_{1-\mathrm{x}} \mathrm{Ca}_{\mathrm{x}} \mathrm{FeO}_{3}$ sensors. Sensors and Actuators 190:25-31 doi: 10.1016/j.snb.2013.08.029

Sun L, He J, Kong H, Yue F, Yang P et al. (2011) Structure, composition and optical properties of $\mathrm{Cu}_{2} \mathrm{ZnSnS}_{4}$ thin films deposited by Pulsed Laser Deposition method. Solar Energy Materials \& Solar Cells 95:2907-2913 doi: 10.1016/j.solmat.2011.06.026

Taelea BM, Narayana H, Mukaro R (2008) Hopping photoconductivity and the effectiveness of phonon detection in GaAs:Zn bolometers. Solid-State Electronics 52:782-786 doi: 10.1016/j.sse.2007.11.013

Todorov TK, Tang J, Bag S, Gunawan O, Gokmen T et al. (2013) Beyond 11\% Efficiency: Characteristics of State-of-the-Art $\mathrm{Cu}_{2} \mathrm{ZnSn}(\mathrm{S}, \mathrm{Se})_{4}$ Solar Cells. Adv. Energy Mater 3:34-38 doi: 10.1002/aenm.201200348

Tsega M, Kuo DH (2013) Characterization and electrical property of the $\mathrm{Cu}$-deficient $\mathrm{Cu}_{2} \mathrm{ZnSn}(\mathrm{S}, \mathrm{Se})_{4}$ bulks at different sulfur contents. Journal of Alloys and Compounds 557:142-146 doi: 10.1016/j.jallcom.2013.01.012 
Wandekar RV, Wani BN, Bharadwaj SR (2009) Crystal Structure, Electrical Conductivity, Thermal Expansion and Compatibility Studies of Co-Substituted Lanthanum Strontium Manganite System. Solid State Sciences 11:240250 doi: 10.1016/j.solidstatesciences.2008.04.018

Conducción hopping en películas nanocristalinas del compuesto CZTSe usado como capa absorbente en celdas solares

Resumen. Se presentan propiedades eléctricas y de transporte en películas nanocristalinas del compuesto cuaternario $\mathrm{Cu}_{2} \mathrm{ZnSnSe}_{4}$ (CZTSe) por método de co-evaporación física. Las muestras fueron crecidas sobre sustratos de vidrio sodalime y variando en rango los parámetros de síntesis: masa de $\mathrm{Cu}$ y temperatura de sustrato. A partir de termopotencia a temperatura ambiente y de transmitancia espectral, se encontró que el material está caracterizado por conductividad tipo $n$ y ancho de banda de energía prohibida de $1.7 \mathrm{eV}$, respectivamente. Las medias de conductividad eléctrica (región de bajas temperaturas; 90-200 K) mostraron que los procesos de conducción se realizan vía hopping de rango de variable entre estados extendidos. Los parámetros que caracterizaron éste mecanismo, energía de activación ( $W_{\text {hopp }}$ ) y rango hopping $\left(\mathrm{R}_{\text {hopp}}\right)$, fueron obtenidos mediante teoría de percolación y modelo difusional. Se obtuvo, que para las muestras CZTSe la densidad de estados de defecto cerca del nivel de Fermi del material, N(EF), está alrededor de 3,403×10 ${ }^{18}$ $\mathrm{cm}^{-3} \mathrm{eV}^{-1}$. Se presentó correlación entre parámetros de depósito y propiedades eléctricas. Se observó influencia de parámetros sobre formación de fases adicionales en el compuesto.

Palabras clave: Hoppingn; propiedades de transporte; semiconductores.
Wei A, Zhao X, Liu J, Zhao Y (2013) Investigation on the structure and optical properties of chemically deposited ZnSe nanocrystalline thin films. Physica B 410:120-125 doi: 10.1016/j.physb.2012.10.031

Condução hopping em películas nano-cristalinas do composto CZTSe utilizado como capa absorvente em painéis solares

Resumo. Apresentam-se propriedades elétricas e de transporte em películas nano-cristalinas do composto quaternário $\mathrm{Cu}_{2} \mathrm{ZnSnSe}_{4}$ (CZTSe) pelo método de co-evaporação física. As amostras foram crescidas sobre substratos de vidro soda-lime e variando a amplitude dos parâmetros de síntese: massa de $\mathrm{Cu}$ e temperatura do substrato. A partir de termo-potência a temperatura ambiente e de transmissão espectral, encontrou-se que o material está caracterizado pela condutividade tipo $n$ e largura de banda de energia proibida de $1.7 \mathrm{eV}$, respetivamente. As medidas de condutividade elétrica (regiões de baixas temperaturas; 90-200 K) mostraram que os processos de condução se realizam via hopping de amplitude variável entre estados estendidos. Os parâmetros que caracterizaram este mecanismo, energia de ativação ( $W_{\text {hopp }}$ ) e amplitude hopping $\left(\mathrm{R}_{\text {hopp}}\right)$, foram obtidos mediante a teoria de percolação e o modelo de difusão. Obteve-se que, para as amostras CZTSe, a densidade de estados de defeito próximos do nível de Fermi do material, $\mathrm{N}\left(\mathrm{E}_{\mathrm{F}}\right)$, está ao redor de, $3,403 \times 10^{18} \mathrm{~cm}^{-3} \mathrm{eV}^{-1}$. Apresentou-se correlação entre parâmetros de depósito e propriedades elétricas. Observou-se influencia de parâmetros sobre a formação de fases no composto.

Palavras-chave: Hopping, propriedades de transporte; semicondutores. 